\title{
Predictors of syncope in patients with severe aortic stenosis: The role of orthostatic unload test
}

\author{
Paweł Kleczyński, Paweł Petkow Dimitrow, Artur Dziewierz, Agata Wiktorowicz, \\ Tomasz Rakowski, Andrzej Surdacki, Dariusz Dudek \\ $2^{\text {nd }}$ Department of Cardiology, Jagiellonian University, Krakow, Poland
}

\begin{abstract}
Background: There is a paucity of data regarding response of cerebral blood flow to the postural unloading maneuver and its impact on the risk of syncope in patients with aortic stenosis (AS). The aim of the present study was to assess effects of orthostatic stress test on changes in carotid and vertebral artery blood flow and its association with syncope in patients with severe AS.

Methods: 108 patients were enrolled (72 with and 36 patients without syncope) with severe isolated severe AS. Peak systolic blood-flow velocity (PSV) and end-diastolic velocity in the carotid arteries and vertebral arteries were measured by duplex ultrasound in the supine position and at 1-2 min after the assumption of the standing position.

Results: The orthostatic stress test induced a significant decrease in carotid and vertebral arterial flow velocities in all examined arteries ( $p<0.001$ ). The median (interquartile range) of mean change in PSV for carotid arteries was higher for patients with syncope (syncope [-] vs. syncope [+]: $-0.6 \mathrm{~cm} / \mathrm{s}$ $[-1.8,1.0]$ vs. $-7.3 \mathrm{~cm} / \mathrm{s}[-9.5,-2.0] ; p<0.001)$ and similarly for vertebral arteries $(-0.5 \mathrm{~cm} / \mathrm{s}[-2.0$, $0.5]$ vs. $-4.8 \mathrm{~cm} / \mathrm{s}[-6.5,-1.3] ; p<0.001$, respectively). Age, aortic valve area, and mean change in $P S V$ for carotid arteries were independently associated with syncope.

Conclusions: In patients with AS, a decrease in carotid and vertebral arterial flow velocities in the standing position was observed and was associated with syncope. The present findings may support the value of an orthostatic test in identifying patients with severe AS and a high risk of syncope. (Cardiol J 2020; 27, 6: 749-755)
\end{abstract}

Key words: aortic stenosis, syncope, orthostatic stress, Doppler ultrasound, cerebral blood flow

\section{Introduction}

Aortic stenosis (AS) with its primarily calcific form has an increasing prevalence in adults of advanced age and therefore requires a comprehensive diagnostic approach and treatment [1]. Syncope is a common symptom in patients with severe AS, and worsens prognosis, however, there remains scarce data regarding its predictors. Patients with severe AS should undergo a Doppler ultrasound examination of carotid and vertebral arteries as a part of a complex assessment before surgical valve replacement or transcatheter aortic valve implantation (TAVI). However, evaluation of valve disorder only at resting conditions may undervalue the real status of the defect and its clinical effects. Orthostatic-induced changes in Doppler echocardiographic evaluation of transaortic gradient in patients with AS have been shown previously [2]. Several studies have reported the importance of an upright position during exercise or in a passive way to determine left ventricular outflow tract gradients in hypertrophic cardiomyopathy and other conditions [3-5]. Moreover, in a recent study a significant blood velocity drop in carotid and vertebral arteries in patients with AS compared to healthy subjects was found but with no relation to syncope, probably due to low sample size [6].

Address for correspondence: Paweł Kleczyński, MD, PhD, $2^{\text {nd }}$ Department of Cardiology, Jagiellonian University, ul. Kopernika 17, 31-501 Kraków, Poland, tel: +48 12424 71 81, fax: +48 12424 71 84, e-mail: kleczu@interia.pl 
In this study, the aim was to assess effects of an orthostatic stress test on changes in carotid and vertebral artery blood flow and its association with syncope in patients with severe AS.

\section{Methods}

One hundred and eight patients who underwent comprehensive echocardiography were included and carotid duplex ultrasound in the documented department from those who fulfilled the following inclusion criteria: severe, "isolated" AS, defined as an aortic valve area $<1.0 \mathrm{~cm}^{2}$; preserved left ventricular ejection fraction $(>50 \%)$; and sinus rhythm. Exclusion criteria were: detected significant atherosclerosis in carotid/vertebral arteries, more than mild concomitant mitral valve dysfunction, or atrial flutter/fibrillation, suboptimal Doppler signal during orthostatic stress. Patients were divided into two groups, patients presenting with (syncope $[+]$ ) and without syncope (syncope [-]). Standard echocardiography was done using Vivid 7 (General Electric, Fairfield, USA). M-mode and two-dimensional echocardiograms were obtained for each patient, which was followed by a pulsed and continuous-wave Doppler ultrasound. Conventional techniques were used to measure the echocardiographic parameters. During the orthostatic test the patient stood for 1-2 min with their left hand on their head and the gradient assessment from the apical window was assessed. The ultrasound transducer $(4-10 \mathrm{MHz}$ linear-array transducer) was used to perform carotid duplex ultrasound routinely in the supine position with peak systolic velocity (PSV) and end diastolic velocity (EDV) assessment in common carotid artery, internal carotid artery (ICA) and vertebral artery (VA). In the second part of the examination, during an orthostatic test the patient stood for 1-2 min and carotid duplex ultrasound was performed, again with velocity measurements, respectively. Heart rate was assessed at baseline and after 1-2 min of orthostatic stress. Additionally, spectral analysis of flow pattern with time-averaged maximum velocity was assessed (TAMAX $[\mathrm{cm} / \mathrm{s}]$ ), time-averaged mean velocity (TAMEAN $[\mathrm{cm} / \mathrm{s}]$ ) and flow volume $[\mathrm{mL} / \mathrm{min}]$ at baseline and in an upright position. Each echocardiographic and Doppler ultrasound parameter was assessed repeatedly 3 times and mean value was taken into the analysis. Change (delta) in PSV and EDV was calculated as a difference between values obtained in the standing and lying position. Mean change of PSV and EDV in carotid arteries was calculated as a mean of measurements from left and right common carotid artery, and left and right ICA. Similarly, mean change of PSV and EDV in vertebral arteries was calculated as a mean of measurements from left and right VA. The study protocol was approved by the institutional ethical board. All patients gave their written consent.

\section{Statistical analysis}

Continuous variables were presented as medians (interquartile ranges). Categorical variables were expressed as percentages. Differences between patients with and without syncope were tested using the $\chi^{2}$ test and the Fisher exact test for dichotomous variables and the Mann-Whitney U-test for continuous variables. Differences between echocardiographic/duplex ultrasound parameters assessed in supine and upright position were assessed using the Wilcoxon signed-rank test. Independent predictors of syncope were identified using multivariate logistic regression analysis. Forward selection in logistic regression with the probability value for covariates to enter the model was set at 0.05 level. All reported clinical, echocardiographic and duplex ultrasound data were tested as possible covariates. Relative risks of syncope were expressed as odds ratios (ORs) with 95\% confidence intervals (CIs). In addition, receiver operating characteristic (ROC) curve analysis was used to assess the ability of selected parameters to detect patients with syncope. Area under ROC curves were compared using DeLong's method. All tests were 2 -tailed, and a p value of $<0.05$ was considered statistically significant. All statistical analysis was performed using SPSS software, version 15.0 (SPSS Inc., Chicago, Illinois).

\section{Results}

A total of 108 patients with severe AS were enrolled. Of these, 72 patients were with and 36 without syncope. Patients with syncope were older and more likely to present with a higher New York Heart Association class and had lower aortic valve area (AVA) as compared with patients without syncope (Table 1). Table 2 depicts Doppler ultrasound data. Median heart rate in patients with syncope was $78.0(65.0-82.0)$ at baseline and $79.0(64.0-83.0)$ in upright position $(\mathrm{p}=0.93)$ and $70.0(60.0-78.0)$ at baseline and $70.0(62.0-79.0)$ while standing in patients without syncope $(\mathrm{p}=0.75)$. The orthostatic stress test induced significant decreases in carotid and vertebral arterial flow velocities ( $p<0.001$ for all). Importantly, change (delta) in PSV and EDV was higher in patients with than without syncope (Table 2). 
Table 1. Demographic, clinical and echocardiographic data.

\begin{tabular}{|c|c|c|c|}
\hline Variable & Syncope (-) (n = 36) & Syncope $(+)(n=72)$ & $\mathbf{P}$ \\
\hline Age [years] & $69.0(64.5,72.0)$ & $79.0(73.5,83.0)$ & $<0.001$ \\
\hline Male gender & $41.7 \%$ & $33.3 \%$ & 0.40 \\
\hline Diabetes mellitus & $25.0 \%$ & $36.1 \%$ & 0.59 \\
\hline NYHA class: & & & $<0.001$ \\
\hline 1 & $47.2 \%$ & $15.3 \%$ & \\
\hline II & $47.2 \%$ & $44.4 \%$ & \\
\hline III & $5.6 \%$ & $37.5 \%$ & \\
\hline IV & $0.0 \%$ & $2.8 \%$ & \\
\hline GFR $\left[\mathrm{mL} / \mathrm{min} / 1.73 \mathrm{~m}^{2}\right]$ & $53.0(45.0,54.0)$ & $58.5(43.8,82.1)$ & 0.56 \\
\hline HR supine [bpm] & $77.0(69.0,80.0)$ & $76.0(65.0,78.5)$ & 0.27 \\
\hline TG max supine $[\mathrm{mmHg}]$ & $95.0(91.0,100.0)$ & $97.0(75.0,107.0)$ & 0.79 \\
\hline TG max upright [mmHg] & $88.0(84.0,90.5)$ & $91.0(70.0,100.0)$ & 0.68 \\
\hline TG max delta [mmHg] & $-7.0(-9.5,-4.0)$ & $-6.0(-9.0,-3.1)$ & 0.29 \\
\hline TG mean supine $[\mathrm{mmHg}]$ & $47.5(44.0,51.0)$ & $50.0(45.0,58.0)$ & 0.11 \\
\hline TG mean upright $[\mathrm{mmHg}]$ & $42.0(40.0,44.0)$ & $47.0(41.0,54.0)$ & 0.002 \\
\hline TG mean delta $[\mathrm{mmHg}]$ & $-4.0(-6.5,-2.0)$ & $-3.0(-4.0,-1.0)$ & 0.049 \\
\hline AVA $\left[\mathrm{cm}^{2}\right]$ & $0.9(0.8,1.0)$ & $0.7(0.6,0.8)$ & $<0.001$ \\
\hline LVEF [\%] & $62.5(60.0,65.0)$ & $62.0(55.0,65.0)$ & 0.54 \\
\hline
\end{tabular}

AVA — aortic valve area; GFR — glomerular filtration rate; HR — heart rate; LVEF — left ventricular ejection fraction; NYHA — New York Heart Association; TG - transaortic gradient

Table 2. Doppler ultrasound data.

\begin{tabular}{lccc}
\hline Variable & Syncope $(-)(\mathbf{n}=36)$ & Syncope $(+)(\mathbf{n}=72)$ & $P$ \\
\hline PSV LCCA [cm/s] & $123.5(117.0,131.5)$ & $100.0(95.0,117.5)$ & $<0.001$ \\
Supine & $124.0(117.0,132.0)$ & $92.0(90.0,116.5)$ & $<0.001$ \\
Upright & $-1.0(-2.5,1.0)$ & $-7.0(-8.0,-2.0)$ & $<0.001$ \\
Delta & & & $<0.001$ \\
EDV LCCA [cm/s] & $22.5(17.0,25.0)$ & $25.0(23.0,27.0)$ & 0.015 \\
Supine & $21.0(17.5,22.5)$ & $22.0(20.0,24.0)$ & 0.014 \\
Upright & $-1.0(-2.5,0.0)$ & $-4.0(-5.0,-1.0)$ & 0.55 \\
Delta & & & 0.017 \\
LCCA TAMAX [cm/s] & $48.2(44.2,49.7)$ & $46.8(43.3,49.6)$ & 0.016 \\
Supine & $45.1(42.2,46.7)$ & $37.8(35.9,44.7)$ & $-6.8(-7.0,-1.2)$ \\
Upright & $-1.8(-3.9,-1.7)$ & & 0.78 \\
Delta & & $23.7(22.1,25.1)$ & 0.029 \\
LCCA TAMEAN [cm/s] & $23.5(22.7,24.8)$ & $18.4(17.4,22.8)$ & 0.024 \\
Supine & $21.2(20.8,22.3)$ & $-4.1(-5.0,-0.7)$ & \\
Upright & $-2.1(-2.6,-1.2)$ & & 0.76 \\
Delta & & $699.9(643.1,723.5)$ & \\
LCCA Flow volume $[\mathrm{mL} / \mathrm{min}]$ & $699.9(636.0,728.1)$ & $674.7(609.2,690.2)$ & \\
Supine & $699.1(629.1,704.2)$ & $-32.3(-42.0,-6.5)$ & \\
Upright & $-25.1(-27.7,-20.7)$ & & 0.06 \\
Delta & & &
\end{tabular}


Table 2 (cont.). Doppler ultrasound data.

\begin{tabular}{|c|c|c|c|}
\hline Variable & Syncope (-) $(n=36)$ & Syncope $(+)(n=72)$ & $\mathbf{P}$ \\
\hline \multicolumn{4}{|c|}{ PSV LICA [cm/s] } \\
\hline Supine & $120.0(113.0,126.5)$ & $96.0(91.0,115.0)$ & $<0.001$ \\
\hline Upright & $122.0(114.5,130.0)$ & $91.0(85.5,115.0)$ & $<0.001$ \\
\hline Delta & $0.0(-2.0,1.5)$ & $-6.0(-9.0,-1.0)$ & $<0.001$ \\
\hline \multicolumn{4}{|c|}{ EDV LICA $[\mathrm{cm} / \mathrm{s}]$} \\
\hline Supine & $20.0(15.0,21.5)$ & $22.0(20.5,23.0)$ & 0.003 \\
\hline Upright & $19.5(15.5,21.0)$ & $20.0(19.0,21.0)$ & 0.049 \\
\hline Delta & $-1.0(-1.0,0.0)$ & $-5.0(-6.0,-1.0)$ & $<0.001$ \\
\hline \multicolumn{4}{|c|}{ PSV LVA [cm/s] } \\
\hline Supine & $45.0(43.0,47.5)$ & $45.0(43.0,49.0)$ & 0.44 \\
\hline Upright & $44.0(42.0,46.0)$ & $40.0(37.5,46.0)$ & 0.01 \\
\hline Delta & $-1.0(-2.0,1.0)$ & $-6.0(-7.0,-1.0)$ & $<0.001$ \\
\hline \multicolumn{4}{|c|}{ EDV LVA [cm/s] } \\
\hline Supine & $9.0(8.0,10.0)$ & $13.0(10.0,15.0)$ & $<0.001$ \\
\hline Upright & $9.0(8.0,10.0)$ & $11.0(10.0,12.0)$ & $<0.001$ \\
\hline Delta & $0.0(-1.0,0.0)$ & $-4.0(-6.0,0.0)$ & 0.001 \\
\hline \multicolumn{4}{|c|}{ PSV RCCA [cm/s] } \\
\hline Supine & $124.0(117.5,132.0)$ & $100.0(93.5,117.5)$ & $<0.001$ \\
\hline Upright & $124.0(117.0,132.0)$ & $94.5(89.5,116.5)$ & $<0.001$ \\
\hline Delta & $-1.0(-3.0,1.0)$ & $-7.0(-9.0,-1.5)$ & 0.001 \\
\hline \multicolumn{4}{|c|}{ EDV RCCA [cm/s] } \\
\hline Supine & $22.5(17.0,25.0)$ & $24.0(22.0,26.0)$ & 0.006 \\
\hline Upright & $21.0(17.5,22.5)$ & $19.0(18.0,23.0)$ & 0.039 \\
\hline Delta & $-1.0(-2.5,0.0)$ & $-4.5(-6.0,-1.0)$ & 0.001 \\
\hline \multicolumn{4}{|c|}{ RCCA TAMAX [cm/s] } \\
\hline Supine & $47.4(44.2,49.7)$ & $46.8(43.3,49.6)$ & 0.72 \\
\hline Upright & $45.0(41.3,47.7)$ & $37.9(36.1,47.5)$ & 0.07 \\
\hline Delta & $-2.1(-3.6,-1.3)$ & $-6.9(-7.1,-1.2)$ & 0.014 \\
\hline \multicolumn{4}{|c|}{ RCCA TAMEAN [cm/s] } \\
\hline Supine & $23.5(22.7,24.7)$ & $23.6(22.2,25.1)$ & 0.64 \\
\hline Upright & $21.3(20.5,22.5)$ & $18.1(17.4,22.3)$ & 0.028 \\
\hline Delta & $-1.8(-2.2,-1.5)$ & $-4.4(-5.2,-0.7)$ & 0.015 \\
\hline \multicolumn{4}{|c|}{ RCCA Flow volume [mL/min] } \\
\hline Supine & $699.9(637.0,728.1)$ & $699.1(641.2,723.7)$ & 0.72 \\
\hline Upright & $687.6(620.8,700.5)$ & $673.5(610.5,710.6)$ & 0.08 \\
\hline Delta & $-20.4(-27.6,-20.9)$ & $-26.8(-28.3,-6.7)$ & $<0.001$ \\
\hline \multicolumn{4}{|c|}{ PSV RICA [cm/s] } \\
\hline Supine & $120.0(113.0,126.5)$ & $95.0(90.5,115.0)$ & $<0.001$ \\
\hline Upright & $122.5(114.5,130.0)$ & $91.5(85.0,115.5)$ & $<0.001$ \\
\hline Delta & $0.0(-2.0,1.5)$ & $-5.8(-7.0,-1.5)$ & $<0.001$ \\
\hline \multicolumn{4}{|c|}{ EDV RICA $[\mathrm{cm} / \mathrm{s}]$} \\
\hline Supine & $20.0(14.5,21.0)$ & $21.5(20.0,23.0)$ & 0.001 \\
\hline Upright & $21.5(16.5,22.0)$ & $18.5(18.5,21.0)$ & 0.03 \\
\hline Delta & $0.0(-1.0,1.0)$ & $-4.5(-6.0,-1.0)$ & $<0.001$ \\
\hline \multicolumn{4}{|c|}{ PSV RVA [cm/s] } \\
\hline Supine & $44.5(43.0,46.5)$ & $45.0(43.0,47.0)$ & 0.52 \\
\hline Upright & $44.0(43.0,46.0)$ & $42.0(40.0,44.5)$ & 0.001 \\
\hline Delta & $0.0(-2.0,1.0)$ & $-5.0(-6.0,-1.0)$ & $<0.001$ \\
\hline \multicolumn{4}{|c|}{ EDV RVA $[\mathrm{cm} / \mathrm{s}]$} \\
\hline Supine & $9.0(8.0,10.0)$ & $13.0(11.0,14.0)$ & $<0.001$ \\
\hline Upright & $9.0(7.5,11.0)$ & $12.0(10.5,12.0)$ & $<0.001$ \\
\hline Delta & $0.0(-1.5,0.0)$ & $-4.0(-6.0,0.0)$ & 0.001 \\
\hline
\end{tabular}

EDV — end diastolic velocity; LCCA — left common carotid artery; LICA — left internal carotid artery; LVA — left vertebral artery; PSV - peak systolic velocity; RCCA — right common carotid artery; RICA — right internal carotid artery; RVA — right vertebral artery; TAMAX — time-averaged maximum velocity; TAMEAN — time-averaged mean velocity 
Table 3. Receiver-operating characteristic curve analysis for the prediction of syncope.

\begin{tabular}{lccccc}
\hline Variable & AUC & P & Optimal cut off value & Sensitivity & Specificity \\
\hline Age [years] & 0.80 & $<0.001$ & $>75$ & $70.8 \%$ & $86.1 \%$ \\
Aortic valve area $\left[\mathrm{cm}^{2}\right]$ & $0.75(0.66-0.83)$ & $<0.001$ & $\leq 0.8$ & $76.5 \%$ & $63.9 \%$ \\
Change in PSV carotid [cm/s] & $0.78(0.68-0.85)$ & $<0.001$ & $\leq-6.0$ & $77.8 \%$ & $77.8 \%$ \\
Change in PSV vertebral [cm/s] & $0.71(0.62-0.79)$ & $<0.001$ & $\leq-3.5$ & $75.0 \%$ & $72.2 \%$ \\
Change in EDV carotid [cm/s] & $0.74(0.65-0.82)$ & $<0.001$ & $\leq-3.3$ & $75.0 \%$ & $72.2 \%$ \\
Change in EDV vertebral [cm/s] & $0.67(0.58-0.76)$ & 0.003 & $\leq-1.5$ & $76.4 \%$ & $52.8 \%$ \\
\hline
\end{tabular}

AUC — area under curve; EDV — end diastolic velocity; PSV — peak systolic velocity

The median (IQR) of mean change in PSV for carotid arteries was higher for patients with syncope (syncope [-] vs. syncope $[+]:-0.6 \mathrm{~cm} / \mathrm{s}$ $(-1.8,1.0)$ vs. $-7.3 \mathrm{~cm} / \mathrm{s}(-9.5,-2.0) ; \mathrm{p}<0.001)$ and similarly for VAs: $(-0.5 \mathrm{~cm} / \mathrm{s}[-2.0,0.5]$ vs. -4.8 $\mathrm{cm} / \mathrm{s}[-6.5,-1.3] ; \mathrm{p}<0.001$, respectively).

In the multivariate logistic regression analysis age (OR 1.101 per 1 year, 95\% CI 1.026-1.183; $\mathrm{p}=0.008)$, AVA (OR 0.071 per $1 \mathrm{~cm}^{2}, 95 \% \mathrm{CI}$ $0.005-1.033 ; \mathrm{p}=0.05)$, mean change in PSV in carotid arteries (OR 0.818 per $1 \mathrm{~cm} / \mathrm{s}, 95 \% \mathrm{CI}$ $0.673-0.995 ; \mathrm{p}=0.044)$ were identified as independent predictors of syncope. In ROC analysis, age, AVA, change in PSV and EDV for both carotid and vertebral arteries were good predictors of syncope (Table 3). However, predictive ability of PSV or EDV derived from carotid arteries was better than what was derived from vertebral arteries.

\section{Discussion}

One hundred and eight patients with severe AS by echocardiography and Doppler ultrasound of blood flow in extracranial arteries in either the supine position or after orthostatic stress were evaluated. Moreover, an assessment to find optimal predictors of syncope in those patients and the ability to identify the following factors predicting syncope: age, AVA and decreased blood flow velocities in carotid and vertebral arteries after an orthostatic unload test. Aortic valve stenosis in its calcific form and with an increasing prevalence in the population of 65 year-olds and over requires a comprehensive clinical and echocardiographic assessment before any treatment [1]. Additionally, patients with severe AS should undergo a Doppler ultrasound examination of carotid and vertebral arteries as a part of a comprehensive assessment before surgical valve replacement or TAVI to reveal any severe stenosis or occlusion. In patients scheduled for TAVI there are concerns linked to the presence of significant stenosis or occlusion of ICA and rapid ventricular pacing during balloon aortic valvuloplasty and prosthesis deployment. In patients undergoing surgery, major concerns are related to intraprocedural hypotonia resulting in hypoperfusion in cerebral blood flow. The common symptom of AS is syncope, which initially may develop only during exercise or with changes in the body position. It may be caused by decreased cerebral blood flow, also as a result of arrhythmias [1, 7-10]. Several studies assessed the increased incidence of syncope in patients with severe AS but failed to show a link between any parameter apart from aortic valve stenosis severity and possible ventricular arrhythmias [9-11]. Left ventricular hypertrophy which occurs in patients with AS and reduced ejection fraction are well-known predictors of higher incidence of arrhythmias [12-15]. Conduction disturbances, resulting with syncope, caused by calcifications are also possible. The evaluation of valve disorder only at resting conditions may underestimate the real state of the defect and its clinical impact. Orthostatic-induced changes in Doppler echocardiographic measures of transaortic gradient in patients with AS have been described before [2]. Standing is an incremental activity of everyday life and may induce a fall in patients predisposed to syncope recognized as important problem in patients with cardiac disease. Performing Doppler ultrasound for screening purposes may not be enough and, in a considered opinion, should also be extended to an orthostatic unload test in AS patients. In a previous study, emphasis of the role of the orthostatic stress test in patients with severe $\mathrm{AS}$, in whom was found a significant decrease in carotid and vertebral arterial flow velocities and flow volume in the upright position [6]. Sato et al. [16] showed that blood flow in the internal carotid artery and medial cerebral artery were reduced 
during the head-up tilt test. Furthermore, Ogoh et al. [17] recently provided data that the effect of graded orthostatic stress on vertebral artery blood flow may be associated with hemodynamic changes in posterior rather than anterior cerebral blood flow. In the present study, however, mean PSV in carotid arteries were highly associated with syncope among all Doppler ultrasound parameters. Moreover, citing another study, the aim was to assess the effects of the orthostatic stress test on carotid and vertebral artery blood flow in patients with severe AS undergoing TAVI [18]. All duplex ultrasound parameters assessed in supine position have significantly improved in patients after TAVI as compared to baseline. The orthostatic stress test induced a decrease in carotid and vertebral arterial flow velocities in AS patients before and after TAVI. However, the drop in velocities and flow volume was numerically lower after TAVI. Therefore, TAVI may have some beneficial effect on extracranial artery blood flow by minimalization of its decrease as a response to orthostatic stress when assessed in long-term follow-up. In another study by Cammalleri et al. [19] monitoring of carotid Doppler measurements may be a useful and noninvasive method for acute assessment of the improvement of hemodynamic flow after TAVI for the cerebral region. A significant improvement of blood flow was found as the systolic peak velocity and the time average mean velocity increased at the end of the procedure.

Orthostatic unload is a very fast, safe and easy stress test without cost. It can be performed without additional equipment in terms of echocardiographic and carotid and vertebral Doppler ultrasound assessment. As shown, the estimation of the changes in cerebral blood flow in response to the orthostatic stress test in patients with AS may be linked to syncope and therefore for prognosis. Moreover, decreased blood flow in carotid and vertebral arteries in the supine position may play a role in asymptomatic patients with AS and may facilitate the time point of a decision about the final treatment - surgical valve replacement or TAVI.

\section{Limitations of the study \\ The exclusion criteria of the study significantly constrained patient recruitment and resulted in relatively small sample size. Quality of acoustic window for Doppler assessment may also limit the use of the method.}

\section{Conclusions}

In patients with AS, a decrease in carotid and vertebral arterial flow velocities in the standing position was observed and was associated with syncope. The present findings may support the value of the orthostatic test in identifying patients with severe AS and a higher risk of syncope.

Conflict of interest: None declared

\section{References}

1. Falk V, Baumgartner H, Bax JJ, et al. ESC Scientific Document Group, ESC Scientific Document Group. 2017 ESC/EACTS Guidelines for the management of valvular heart disease. Eur Heart J. 2017; 38(36): 2739-2791, doi: 10.1093/eurheartj/ehx391, indexed in Pubmed: 28886619.

2. Dimitrow PP, Sorysz D. Orthostatic stress echocardiography as a useful test to measure variability of transvalvular pressure gradients in aortic stenosis. Cardiovasc Ultrasound. 2013; 11: 15, doi: 10.1186/1476-7120-11-15, indexed in Pubmed: 23706028.

3. Dimitrow PP, Bober M, Michałowska J, et al. Left ventricular outflow tract gradient provoked by upright position or exercise in treated patients with hypertrophic cardiomyopathy without obstruction at rest. Echocardiography. 2009; 26(5): 513-520, indexed in Pubmed: 19452607.

4. Dimitrow PP, Cheng TO. Standing position alone or in combination with exercise as a stress test to provoke left ventricular outflow tract gradient in hypertrophic cardiomyopathy and other conditions. Int J Cardiol. 2010; 143(3): 219-222, doi: 10.1016/j. ijcard.2010.04.026, indexed in Pubmed:20442001.

5. Mason DT, Braunwald E, Ross J. Effects of changes in body position on the severity of obstruction to left ventricular outflow in idiopathic hypertrophic subaortic stenosis. Circulation. 1966; 33(3): 374-382, indexed in Pubmed: 5904414.

6. Kleczyński P, Petkow Dimitrow P, Dziewierz A, et al. Decreased carotid and vertebral arterial blood-flow velocity in response to orthostatic unload in patients with severe aortic stenosis. Cardiol J. 2016; 23(4): 393-401, doi: 10.5603/CJ.a2016.0043, indexed in Pubmed: 27367481.

7. Ross J. Jr, Braunwald E. Aortic stenosis Circulation. 1968; 38: 61-67.

8. Schwartz LS, Goldfischer J, Sprague GJ, et al. Syncope and sudden death in aortic stenosis. Am J Cardiol. 1969; 23(5): 647-658, indexed in Pubmed:5771033.

9. Satoh M, Saeki M, Yamazoe M, et al. Syncope in aortic stenosis during continuous electrocardiographic monitoring. A case report. Jpn Circ J. 1988; 52(12): 1415-1418, doi: 10.1253/ jcj.52.1415.

10. Omran H, Fehske W, Rabahieh R, et al. Valvular aortic stenosis: risk of syncope. J Heart Valve Dis. 1996; 5(1): 31-34, indexed in Pubmed: 8834722 .

11. Orłowska-Baranowska E, Baranowski R, Hryniewiecki T. Incidence of syncope and cardiac arrest in patients with severe aortic stenosis. Pol Arch Med Wewn. 2014; 124(6): 306-312, indexed in Pubmed: 24781653.

12. Sorgato A, Faggiano P, Aurigemma GP, et al. Ventricular arrhythmias in adult aortic stenosis: prevalence, mechanisms, 
and clinical relevance. Chest. 1998; 113(2): 482-491, indexed in Pubmed: 9498969.

13. Batur MK, Açil T, Onalan O, et al. Is ventricular repolarization heterogeneity a cause of serious ventricular tachyarrhythmias in aortic valve stenosis? Clin Cardiol. 2000; 23(6): 449-452, indexed in Pubmed: 10875037.

14. Koşar F, Tandoğan I, Hisar I, et al. QTc dispersion measurement for risk of syncope in patients with aortic stenosis. Angiology. 2001; 52(4): 259-265, doi: 10.1177/000331970105200405, indexed in Pubmed: 11330508.

15. Tsai JP, Lee PY, Wang KT, et al. Torsade de pointes in severe aortic stenosis: case report. J Heart Valve Dis. 2007; 16(5): 504-507, indexed in Pubmed:17944122.

16. Sato K, Fisher J, Seifert T, et al. Blood flow in internal carotid and vertebral arteries during orthostatic stress. Exp Physiol. 2012; 97(12): 1272-1280, doi:10.1113/expphysiol.2012.064774.
17. Ogoh S, Sato K, Okazaki K, et al. Blood flow in internal carotid and vertebral arteries during graded lower body negative pressure in humans. Exp Physiol. 2015; 100(3): 259-266, doi: 10.1113/expphysiol.2014.083964, indexed in Pubmed: 25641216.

18. Kleczyński P, Petkow Dimitrow P, Dziewierz A, et al. Transcatheter aortic valve implantation improves carotid and vertebral arterial blood flow in patients with severe aortic stenosis: practical role of orthostatic stress test. Clin Cardiol. 2017; 40(7): 492-497, doi: 10.1002/clc.22684, indexed in Pubmed: 28273361.

19. Cammalleri V, Romeo F, Marchei M, et al. Carotid Doppler sonography: additional tool to assess hemodynamic improvement after transcatheter aortic valve implantation. J Cardiovasc Med (Hagerstown). 2018; 19(3): 113-119, doi: 10.2459/JCM.0000000000000622, indexed in Pubmed: 29351134. 